Ergod. Th. \& Dynam. Sys. (1982), 2, 465-489

Printed in Great Britain

\title{
Factors of horocycle flows
}

\author{
MARINA RATNER \\ Department of Mathematics, University of California at Berkeley, \\ Berkeley, Calif. 94720, USA
}

(Received 7 October 1981)

\section{Dedicated to the memory of V. M. Alexeyev}

Abstract. We classify up to an isomorphism all factors of the classical horocycle flow on the unit tangent bundle of a surface of constant negative curvature with finite volume.

Let $T=\left\{T_{t}, t \in R\right\}$ and $S=\left\{S_{t}, t \in R\right\}$ be two measure preserving (m.p.) flows on probability spaces $(X, \mu)$ and $(Y, \nu)$ respectively. We say that $S$ is a factor of $T$ if there is a measure preserving

$$
\psi: X \rightarrow Y \quad \text { such that } \psi\left(T_{t} x\right)=S_{t} \psi(x)
$$

for all $t \in R$ and $\mu$-almost every (a.e.) $x \in X$. $\psi$ is called a conjugacy between $T$ and $S . T$ and $S$ are called isomorphic $(T \sim S)$ if there is an invertible conjugacy between $T$ and $S$, called an isomorphism. We write $(T, S) \sim\left(T^{\prime}, S^{\prime}\right)$ if $T \sim T^{\prime}$ and $S \sim S^{\prime} . S$ is called trivial if there is $y \in Y$ such that $\nu\{y\}=1$. Henceforth the word 'factor' means non-trivial factor.

Let $\Phi(T)$ denote the set of all isomorphisms

$$
\phi: X \rightarrow X \quad \text { such that } \phi\left(T_{t} x\right)=T_{t} \phi(x)
$$

for all $t \in R$ and a.e. $x \in X$ and let $\Psi=\Psi(T, S)$ denote the set of all conjugacies between $T$ and $S$. We say that $\psi_{1} \in \Psi$ and $\psi_{2} \in \Psi$ are equivalent $\left(\psi_{1} \sim \psi_{2}\right)$ if there are $\phi_{1} \in \Phi(T)$ and $\phi_{2} \in \Phi(S)$ such that $\psi_{2}=\phi_{2} \circ \psi_{1} \circ \phi_{1}$ a.e.

Let $\pi(T, S)$ denote the set of equivalence classes in $\Psi$. It is clear that if $(T, S) \sim$ $\left(T^{\prime}, S^{\prime}\right)$ then there is a natural one-to-one correspondence between $\pi(T, S)$ and $\pi\left(T^{\prime}, S^{\prime}\right)$. So $|\pi(T, S)|$ is an invariant of the isomorphism class of $(T, S)$.

One would naturally raise the following problems: (1) classifying all possible factors of a given m.p. flow $T$ up to an isomorphism; (2) describing $\pi(T, S)$ for a given factor $S$ of $T$.

In this paper we shall solve these problems for the classical horocycle flow on the unit tangent bundle of a surface of constant negative curvature with finite volume.

Let $G$ denote the group $\operatorname{SL}(2, R)$ equipped with a left invariant Riemannian metric and let $\mathscr{T}$ be the set of all discrete subgroups $\Gamma$ of $G$ such that the quotient space $M=\Gamma \backslash G=\{\Gamma g: g \in G\}$ has finite volume. $M$ can be viewed as the unit tangent bundle of a surface of constant negative curvature with finite volume. Let $F$ be an element of the Lie algebra $\mathscr{A}$ of $G$ and let $F_{t}=\exp (t F) \in G$. The flow $f=\left\{f_{t}, t \in R\right\}$ 
on $M$ defined by $f_{t}(\Gamma g)=\Gamma g \cdot F_{t}, g \in G, t \in R$ is called the algebraic flow, generated by $F . f$ preserves the Riemannian volume $v$ on $M$ derived from the Haar measure on $G . v$ is defined on the Borel $\sigma$-algebra $B_{M}$ of $M$ and we denote by $(\mathscr{B}, \mu)$ the normalized completion of $\left(B_{M}, v\right), \mu(M)=1$.

The horocycle flow

$$
h=\left\{h_{t}, t \in R\right\}
$$

on $M$ is the algebraic flow, generated by $\left(\begin{array}{ll}0 & 0 \\ 1 & 0\end{array}\right)$, i.e.

$$
h_{t}(\Gamma g)=\Gamma g N_{t}
$$

where

$$
N_{t}=\left(\begin{array}{ll}
1 & 0 \\
t & 1
\end{array}\right), \quad t \in R, g \in G .
$$

It is well known that $h$ is ergodic and mixing on $(M, \mu)$, in fact mixing of all degrees [1].

Let $F \in \mathscr{A}, \Gamma_{i} \in \mathscr{T}, i=1,2$ and let $f^{(i)}$ be the algebraic flow on $M_{i}=\Gamma_{i} \mid G$, generated by $F, i=1,2$. It is easy to see that if $\Gamma_{1} \subset \Gamma_{2}$ then $f^{(2)}$ is a factor of $f^{(1)}$. Indeed, let

$$
\psi: M_{1} \rightarrow M_{2}
$$

be defined by

$$
\psi\left(\Gamma_{1} g\right)=\Gamma_{2} g, \quad g \in G
$$

Then $\psi$ is measure preserving and

$$
\psi f_{t}^{(1)}\left(\Gamma_{1} g\right)=\psi\left(\Gamma_{1} g F_{t}\right)=\Gamma_{2} g F_{t}=f_{t}^{(2)}\left(\Gamma_{2} g\right)=f_{t}^{(2)}\left(\psi\left(\Gamma_{1} g\right)\right)
$$

We shall call $f^{(2)}$ an algebraic factor of $f^{(1)}$.

The following theorem shows that every factor of the horocycle flow is algebraic.

THEOREM 1. Let $\Gamma_{1} \in \mathscr{T}, M_{1}=\Gamma_{1} \backslash G$ and let $S$ be a factor of the horocycle $h^{(1)}$ on $\left(M_{1}, \mu_{1}\right)$. Then there is $\Gamma_{2} \in \mathscr{T}$ such that $\Gamma_{1} \subset \Gamma_{2}$ and $S$ is isomorphic to $h^{(2)}$ on $\left(M_{2}, \mu_{2}\right)$.

It has been proved in [4] that for $\Gamma_{1}, \Gamma_{2} \in \mathscr{T}$ the horocycle flows $h^{(1)}$ and $h^{(2)}$ are isomorphic iff $\Gamma_{1}$ and $\Gamma_{2}$ are conjugate in $G$, i.e. $\Gamma_{2}=C \Gamma_{1} C^{-1}$ for some $C \in G$. For $\Gamma \in \mathscr{T}$ we denote

$$
\alpha(\Gamma)=\{\tilde{\Gamma} \in \mathscr{T}: \Gamma \subset \tilde{\Gamma}\} .
$$

It is well known [6] that $\alpha(\Gamma)$ is finite. $\Gamma$ is called maximal if $\alpha(\Gamma)=\{\Gamma\}$. We get the following corollary.

COROLlARY 1. The number of non-isomorphic factors of the horocycle flow $h$ on $M=\Gamma \backslash G, \Gamma \in \mathscr{T}$ is finite and equals the number of conjugacy classes in $\alpha(\Gamma)$.

It was proved in [4] that if $\Gamma_{2} \in \alpha\left(\Gamma_{1}\right)$ and $\psi: M_{1} \rightarrow M_{2}$ is a conjugacy between $h^{(1)}$ and $h^{(2)}$ then there is $C \in G$ such that

$$
C \Gamma_{1} C^{-1} \subset \Gamma_{2} \quad \text { and } \quad \psi\left(\Gamma_{1} g\right)=h_{\sigma}^{(2)} \psi_{C}\left(\Gamma_{1} g\right)
$$

for some $\sigma \in R$ and a.e. $\Gamma_{1} g \in M_{1}, g \in G$, where $\psi_{C}\left(\Gamma_{1} g\right)=\Gamma_{2} C g$. This says that $\psi \sim \psi_{C}$. 
For $\Gamma_{2} \in \alpha\left(\Gamma_{1}\right)$ we denote

$$
\mathscr{C}\left(\Gamma_{1}, \Gamma_{2}\right)=\left\{C \in G: C \Gamma_{1} C^{-1} \subset \Gamma_{2}\right\}=\left\{C \in G: C^{-1} \Gamma_{2} C \in \alpha\left(\Gamma_{1}\right)\right\}
$$

and

$$
\kappa\left(\Gamma_{1}, \Gamma_{2}\right)=\left\{\Gamma \in \alpha\left(\Gamma_{1}\right): \Gamma=C^{-1} \Gamma_{2} C \text { for some } C \in G\right\} .
$$

It follows from [4] that

$$
\psi_{C_{1}} \sim \psi_{C_{2}}, \quad C_{1}, C_{2} \in \mathscr{C}\left(\Gamma_{1}, \Gamma_{2}\right) .
$$

iff $C_{2}=C C_{1} D$ for some $C \in \tilde{\Gamma}_{2}$ and some $D \in \tilde{\Gamma}_{1}$, where $\tilde{\Gamma}$ denotes the normalizer of $\Gamma$ in $G$, i.e.

$$
\tilde{\Gamma}=\left\{C \in G: C \Gamma C^{-1}=\Gamma\right\} .
$$

In this case we write $C_{2} \sim C_{1}$. $\sim$ is an equivalence relation in $\mathscr{C}\left(\Gamma_{1}, \Gamma_{2}\right)$. For $\Gamma^{\prime}, \Gamma^{\prime \prime} \in \kappa\left(\Gamma_{1}, \Gamma_{2}\right)$ we write $\Gamma^{\prime} \sim \Gamma^{\prime \prime}$ if $\Gamma^{\prime \prime}=D^{-1} \Gamma^{\prime} D$ for some $D \in \tilde{\Gamma}_{1}$. It is clear that $C_{2} \sim C_{1}$ in $\mathscr{C}\left(\Gamma_{1}, \Gamma_{2}\right)$ iff $C_{2}^{-1} \Gamma_{2} C_{2} \sim C_{1}^{-1} \Gamma_{2} C_{1}$ in $\kappa\left(\Gamma_{1}, \Gamma_{2}\right)$. We have just proved the following theorem.

THEOREM 2. Let $\Gamma_{1}, \Gamma_{2} \in \mathscr{T}$ and $\Gamma_{1} \subset \Gamma_{2}$. Then

$$
\pi\left(h^{(1)}, h^{(2)}\right)=\left\{\left[\psi_{C}\right]: C \in \mathscr{C}\left(\Gamma_{1}, \Gamma_{2}\right)\right\}
$$

where $[\psi]$ denotes the equivalence class of $\psi \in \Psi\left(h^{(1)}, h^{(2)}\right), \pi\left(h^{(1)}, h^{(2)}\right)$ is finite and $\left|\pi\left(h^{(1)}, h^{(2)}\right)\right|$ equals the number of equivalence classes in $\kappa\left(\Gamma_{1}, \Gamma_{2}\right)$.

COROLlary 2. If $\Gamma$ is maximal and $S$ is a factor of $h$ on $\Gamma \backslash G$, then $S$ is isomorphic to $h$ and $|\pi(h, S)|=1$.

THEOREM 3. Let $S$ on $(Y, \nu)$ be a factor of $h_{1}$ (the time-one transformation of the horocycle flow) on $(M=\Gamma \backslash G, \mu), \Gamma \in \mathscr{T}$ with a conjugacy $\psi: M \rightarrow Y, \psi h_{1}(x)=h_{1} \psi(x)$ a.e. $x \in M$. Then there exists a m.p. flow $\left\{S_{t}, t \in R\right\}$ on $(Y, \nu)$ such that $S=S_{1}$ and $\psi h_{t}(x)=S_{t} \psi(x)$ for all $t \in R$ and a.e. $x \in M$.

COROLlaRy 3. If $S$ is a factor of $h_{1}^{(1)}$ on $M_{1}=\Gamma_{1} \backslash G$ then there is $\Gamma_{2} \supset \Gamma_{1}$ such that $S$ is isomorphic to $h_{1}^{(2)}$ on $M_{2}=\Gamma_{2} \backslash G$. If $\Gamma_{1}$ is maximal then every factor of $h_{1}^{(1)}$ is isomorphic to $h_{1}^{(1)}$.

The geodesic flow $g=\left\{g_{t}, t \in R\right\}$ on $M=\Gamma \backslash G, \Gamma \in \mathscr{T}$ is the algebraic flow, generated by $\left(\begin{array}{rr}1 & 0 \\ 0 & -1\end{array}\right) \in \mathscr{A}$, i.e.

$$
g_{t}(\Gamma x)=\Gamma x\left(\begin{array}{cc}
\exp (t) & 0 \\
0 & \exp (-t)
\end{array}\right), \quad x \in G .
$$

$g$ and $h$ satisfy the following commutation relation:

$$
g_{\ddagger} \circ h_{s}=h_{s \exp (2 t)} \circ g_{t}, \quad t, s \in R .
$$

(*) shows that $h_{\alpha}$ and $h_{\beta}$ are isomorphic if $\alpha \cdot \beta>0$ and that the entropy of $h$ is zero. 
It is well known that $g$ is Bernoulli [2] and therefore $g$ has uncountably many non-isomorphic factors. $(*)$ shows that the entropy of $g$ equals 2 for every $\Gamma \in \mathscr{T}$. This implies that $g^{(1)}$ is isomorphic to $g^{(2)}$ for any $\Gamma_{1}, \Gamma_{2} \in \mathscr{T}$. One can show that $\pi\left(g^{(1)}, g^{(2)}\right)$ is uncountable.

The proof of theorem 1 consists of three basic steps: (1) We show ( $\$ 3$ ) that if a flow $S$ on $(Y, \nu)$ is a factor of the horocycle flow $h$ on $(M, \mu)$ with a factor map $\psi: M \rightarrow Y$ then $\psi^{-1}\{y\}$ is finite for a.e. $y \in Y$. This uses the basic estimates on divergence of horocycles $(\$ 2)$ to show that $\psi$ is locally $1-1 ;(2)$ using (1) we show that any factor map of the horocycle flow must be a factor map of the entire action of $\operatorname{SL}(2, R)(\$ 4)$; (3) using (2), we construct a discrete subgroup of $\operatorname{SL}(2, R)$ for which the factor is a horocycle flow (the end of $\$ 4$ ).

Section 1 contains some measure-theoretical background and in $\S 5$ we prove theorem 3 .

I am grateful to Joe Wolf for valuable discussions.

\section{Factors and invariant partitions}

Henceforth all measure spaces are assumed to be separable and complete.

Let $S=\left\{S_{t}, t \in R\right\}$ on $(Y, \nu)$ be a factor of $T=\left\{T_{t}, t \in R\right\}$ on $(X, \mu)$ with a conjugacy $\psi: X \rightarrow Y$

$$
\psi T_{t}(x)=S_{t} \psi(x) \text { for all } t \in R \text { and a.e. } x \in X
$$

We can assume without loss of generality that (1.1) holds for all $x \in X$. $\psi$ induces a measurable partition

$$
\xi=\xi(\psi)=\left\{\psi^{-1}\{y\}: y \in Y\right\}
$$

of $X$ (see [5]), invariant under $T$, i.e. for every $t \in R$

$$
C \in \xi \quad \text { iff } T, C \in \xi \text {. }
$$

Let $X / \xi$ be the quotient space, induced by $\xi$ and let $\pi: X \rightarrow X / \xi$ be the projection $\pi(x)=C(x)$, where $C(x)$ denotes the atom of $\xi$, containing $x$. A set $A \subset X / \xi$ is called measurable in $X / \xi$ if $\pi^{-1}(A)$ is measurable in $X$. We define a measure $\mu_{\xi}$ on $X / \xi$ by $\mu_{\xi}(A)=\mu\left(\pi^{-1}(A)\right)$. $\pi$ is a conjugacy between $T$ and the m.p. flow $T^{\xi}$ on $X / \xi$ defined by

$$
T_{t}^{\xi}(C(x))=C\left(T_{t} x\right), \quad x \in X, t \in R .
$$

It is clear, that $T^{\xi}$ is isomorphic to $S$.

It is well known (see [5]) that for a.e. $C \in \xi$ there is a probability measure $\mu_{C}$ on $C$ such that if $A \subset X$ is measurable in $X$ then $A \cap C$ is measurable in $C$ and

$$
\mu(A)=\int_{X / \xi} \mu_{C}(A \cap C) d \mu_{\xi}(C) .
$$

Henceforth it will be clear from the context when $C \in \xi$ is considered as a subset of $X$ and when it is considered as a point of $X / \xi$. The family of measures $\left\{\mu_{C}\right\}$ is unique in the following sense: a family $\left\{\mu_{C}^{\prime}\right\}$ satisfies (1.2) iff $\mu_{C}^{\prime}=\mu_{C}$ for a.e. 
$C \in X / \xi$. This says that by possibly changing $\left\{\mu_{C}\right\}$ on a set of $\mu_{\xi}$-measure zero we can get a set

$$
\Omega \subset X / \xi, \quad T_{t}^{\xi} \Omega=\Omega, \quad t \in R, \quad \mu_{\xi}(\Omega)=1
$$

such that if $C \in \Omega$ then

$$
\begin{aligned}
& A \subset C \text { is measurable in } C \text { iff } T_{t} A \text { is measurable in } T_{t} C \\
& \text { and } \mu_{C}(A)=\mu_{T_{t} C} T_{t} A \text { for all } t \in R .
\end{aligned}
$$

We can assume without loss of generality that (1.3) holds for all $C \in X / \xi$, since $T^{\xi}$ restricted on $\Omega$ is isomorphic to $T^{\xi}$ on $X / \xi$.

We say that $\mu_{C}$ is atomic if there is $x \in C$ s.t. $\mu_{C}\{x\}>0$.

Proposition 1.1. Suppose that $T$ is ergodic and that there is $Z \subset X / \xi, \mu_{\xi}(Z)>0$ such that $\mu_{C}$ is atomic for every $C \in Z$. Then there are

$$
\begin{array}{cl}
U \subset X / \xi, & T_{t}^{\xi} U=U, \quad t \in R, \quad \mu_{\xi}(U)=1, \\
D \subset X, & T, D=D, \quad t \in R, \quad \mu(D)=1
\end{array}
$$

and an integer $n>0$ such that for every $C \in U, D \cap C$ consists of exactly $n$ points $x_{1}(C), \ldots, x_{n}(C)$ with

$$
\mu_{C}\left\{x_{i}(C)\right\}=\frac{1}{n}, \quad i=1, \ldots, n .
$$

Proof. Let $m: X / \xi \rightarrow R$ be defined by

$$
m(C)=\sup \left\{\mu_{C}\{x\}: x \in C\right\} .
$$

$m$ is measurable [5] and (1.3) shows that $m$ is constant on orbits of $T^{\xi}$. Since $T^{\xi}$ is ergodic, there is

$$
U^{\prime} \subset X / \xi, \quad T_{l}^{\xi} U^{\prime}=U^{\prime}, \quad t \in R, \quad \mu_{\xi}\left(U^{\prime}\right)=1
$$

such that $m$ equals a constant $\alpha$ on $U^{\prime}$. Since

$$
\mu_{\xi}\left(Z \cap U^{\prime}\right)>0 \text { and } m(C)>0
$$

for every $C \in Z, \alpha$ must be positive.

Let

$$
D=\left\{x \in X: C(x) \in U^{\prime} \text { and } \mu_{C}\{x\}=\alpha\right\} .
$$

$D$ is measurable [5] and (1.3) shows that $D$ consists of orbits of $T$. It is clear, that $\mu(D)>0$. Since $T$ is ergodic, $\mu(D)=1$.

Let

$$
\begin{gathered}
U=\left\{C \in U^{\prime}: \mu_{C}(C \cap D)=1\right\}, \\
\mu_{\xi}(U)=1, \quad T_{t}^{\xi} U=U, \quad t \in R .
\end{gathered}
$$

If $x \in C \cap D$ then $\mu_{C}\{x\}=\alpha>0, C \in U$. This says that $C \cap D, C \in U$ consists of finite many points $x_{1}(C), \ldots, x_{n}(C)$ and that $\alpha=1 / n$, since $\mu_{C}(C \cap D)=1, C \in U$. This completes the proof. 
It also follows from [5] that if a.e. $C \in X / \xi$ consists of $n$ points of equal $\mu_{C}$-measure, then there are a measurable

$$
V \subset X / \xi, \quad \mu_{\xi}(V)=1, \quad \pi^{-1}(V)=\tilde{X}, \quad \mu(\tilde{X})=1
$$

and pairwise disjoint measurable $X_{i} \subset X, i=1, \ldots, n$,

$$
X=\bigcup_{i=1}^{n} X_{i}, \quad \mu\left(X_{i}\right)=\frac{1}{n}, \quad i=1, \ldots, n
$$

such that if $C \in V$ then

$$
C \cap X_{i}=\left\{x_{i}(C)\right\}
$$

consists of exactly one point and the maps $\phi_{i}: \tilde{X}$ onto $X_{i}$ defined by

$$
\phi_{i}(x)=x_{i}(C(x))
$$

are measurable, $i=1, \ldots, n$. The pair $\left(X_{i}, \phi_{i}\right)$ is called a measurable cross-section of $\xi, i=1, \ldots, n$.

2. Properties of the covering horocycle flow in $G$

Let $p: G \rightarrow M=\Gamma \backslash G, \Gamma \in \mathscr{T}$ be the covering projection $p(g)=\Gamma g$. Let

$$
G_{t} g=g \cdot\left(\begin{array}{cc}
e^{t} & 0 \\
0 & e^{-t}
\end{array}\right) \quad \text { and } H_{t} g=g \cdot\left(\begin{array}{ll}
1 & 0 \\
t & 1
\end{array}\right) \quad g \in G, t \in R
$$

be the geodesic and the horocycle flows on $G$, covering $\left\{g_{t}\right\}$ and $\left\{h_{t}\right\}$ on $M$ respectively. We shall also consider the flow

$$
H_{t}^{*} g=g \cdot\left(\begin{array}{ll}
1 & t \\
0 & 1
\end{array}\right)
$$

on $G$, covering the flow

$$
h_{\imath}^{*}(\Gamma g)=\Gamma H_{\imath}^{*} g
$$

on $M$.

We have

$$
\begin{aligned}
G_{t} \circ H_{s} & =H_{s \exp (2 t)} \circ G_{t} \\
G_{t} \circ H_{s}^{*} & =H_{s \exp (-2 t)}^{*} \circ G_{t}, \quad t, s \in R .
\end{aligned}
$$

We assume that $G$ is equipped with a left invariant Riemannian metric, in which the length of the orbit intervals $\left[g, G_{t} g\right],\left[g, H_{t} g\right]$ and $\left[g, H_{i}^{*} g\right]$ is $t, g \in G$. Let $d: G \times G \rightarrow R^{+}$be the left invariant metric on $G$, induced by this Riemannian metric and let $e$ denote the identity element of $G$.

Denote

$$
\Delta(g)=\max \{|1-a|,|b|,|c|\} \quad \text { for } g=\left(\begin{array}{ll}
a & b \\
c & d
\end{array}\right) \in G .
$$

It is well known, that there is $A>1$ such that

$$
A^{-1} \Delta(g) \leq d(e, g) \leq A \Delta(g) \text { for all } g \in G \text { with } d(g, e) \leq 1 .
$$

For $x, y \in G$ we have

$$
d\left(H_{s} x, H_{s} y\right)=d\left(e, N_{-s} \cdot g \cdot N_{s}\right)
$$


where $g=x^{-1} \cdot y$ and $N_{s}=\left(\begin{array}{ll}1 & 0 \\ s & 1\end{array}\right)$. It follows from (2.2) that if $d\left(H_{s} x, H_{s} y\right) \leq 1$, then

$$
A^{-1} \Delta\left(N_{-s} \cdot g \cdot N_{s}\right) \leq d\left(H_{s} x, H_{s} y\right) \leq A \Delta\left(N_{-s} \cdot g \cdot N_{s}\right)
$$

where.

and

$$
\Delta\left(N_{-s} \cdot g \cdot N_{s}\right)=\max \left\{|1-a-b s|,|b|,\left|b s^{2}+s(a-d)-c\right|\right\}
$$

$$
g=x^{-1} \cdot y=\left(\begin{array}{ll}
a & b \\
c & d
\end{array}\right) \text {. }
$$

Let $0<\varepsilon \leq 1$ be small and suppose that $d(x, y)<\varepsilon$. We shall now estimate the length of the time the horocycle orbits $H_{t} x$ and $H_{t} y$ stay within $\varepsilon$. (2.3) shows that $d\left(H_{s} x, H_{s} y\right)$ grows polynomially in $s$. We have

$$
\left\{s \in R^{+}: d\left(H_{s} x, H_{s} y\right) \leq \varepsilon\right\} \subset\left\{s \in R^{+}: \Delta\left(N_{-s} \cdot g \cdot N_{s}\right) \leq A \cdot \varepsilon\right\}=E(g, \varepsilon)
$$

where $g=x^{-1} \cdot y$ and $\Delta\left(N_{-s} \cdot g \cdot N_{s}\right)$ are as in (2.3).

It is easy to compute that:

(1) $E(g, \varepsilon)$ consists of at most two connected components $E_{0}=E_{0}(g, \varepsilon) \ni 0$ and $E_{1}=E_{1}(g, \varepsilon)$;

(2) If

$$
l=l(g, \varepsilon)=\max \left\{l\left(E_{0}\right), l\left(E_{1}\right)\right\} \geq 1(l(I) \text { denotes the length of } I),
$$

then for every $s \in E(g, \varepsilon)$ we have

$$
\left|1-a_{s}\right| \leq D(\varepsilon) / l, \quad\left|b_{s}\right| \leq D(\varepsilon) / l^{2}, \quad\left|c_{s}\right| \leq \varepsilon
$$

where

$$
\left(\begin{array}{ll}
a_{s} & b_{s} \\
c_{s} & d_{s}
\end{array}\right)=N_{-s} \cdot g \cdot N_{s} \quad \text { and } \quad \varepsilon \leq D(\varepsilon) \rightarrow 0
$$

when $\varepsilon \rightarrow 0$.

It follows from (2.3) and (2.5) that if $l \geq 1$ then

$$
\Delta\left(N_{-s-u} \cdot g \cdot N_{s+u}\right) \leq 3 D(\varepsilon) \text { for all } s \in E(g, \varepsilon) \text { and all } 0 \leq u \leq l .
$$

This implies that

$$
d\left(H_{s+u} x, H_{s+u} y\right) \leq 3 A D(\varepsilon) \text { for all } s \in E(g, \varepsilon) \text { and all } 0 \leq u \leq l .
$$

Henceforth $D(\varepsilon)$ will always mean a constant depending only on $\varepsilon$ and converging to 0 when $\varepsilon \rightarrow 0$.

Let us observe that if

$$
g=\left(\begin{array}{ll}
a & b \\
c & d
\end{array}\right), \quad \Delta(g)<\varepsilon
$$

and $\varepsilon$ is sufficiently small then

$$
g=H_{q} H_{r}^{*} G_{p} e \text { where } p=\log a /(1+b c), \quad r=b e^{-p}, \quad q=c e^{p} .
$$


For $g \in G$ and $\alpha, \beta, \gamma \geq 0$ we define

$$
U(g ; \alpha, \beta, \gamma)=\left\{\tilde{g} \in G: \tilde{g}=H_{q} H_{r}^{*} G_{p} g \text { for some }|p| \leq \alpha,|r| \leq \beta,|q| \leq \gamma\right\} .
$$

It follows from (2.1) that for every $t \in R$

$$
G_{t} U(g ; \alpha, \beta, \gamma)=U\left(G_{t} g ; \alpha, \beta e^{-2 t}, \gamma e^{2 t}\right) .
$$

It follows from (2.4), (2.5) and (2.7) that if $s \in E\left(x^{-1} \cdot y, \varepsilon\right)$ and $l=l\left(x^{-1} \cdot y, \varepsilon\right) \geq 1$ then

$$
H_{s} y \in U\left(H_{s} x, D(\varepsilon) / l, D(\varepsilon) / l^{2}, D(\varepsilon)\right)
$$

where $D(\varepsilon) \rightarrow 0$ when $\varepsilon \rightarrow 0$.

We shall need the following:

LEMMA 2.1. Given $0<\delta<1$ there are $\tilde{\delta}>0$ and $\bar{\delta}>0$ depending only on $\delta$ such that if $d(x, y)<\tilde{\delta}, x, y \in G$ then for every $s \in E\left(x^{-1} \cdot y, 1\right)$ and every $0 \leq u<$ $\overline{\delta l}\left(x^{-1} \cdot y, 1\right)$ with $s+u \notin E\left(x^{-1} y, 1\right)$

$$
\text { either } d\left(H_{s+u} x, H_{s+u+1} y\right)<\delta \text { or } d\left(H_{s+u} x, H_{s+u-1} y\right)<\delta .
$$

Proof. It is enough to show that there are $\tilde{\delta}>0$ and $\bar{\delta}>0$ such that if $\Delta(g)<\tilde{\delta}$, $g \in G$ then for every $s \in E(g, 1)$ and every

$$
0 \leq u \leq \bar{\delta} l(g, 1) \text { and } s+u \notin E(g, 1)
$$

we have $\left|c_{s+u}\right|>1$ and

$$
\max \left\{\left|1-a_{s+u}\right|,\left|b_{s+u}\right|,\left|c_{s+u}-\operatorname{sign} c_{s+u}\right|\right\} \leq \delta,
$$

where

$$
g_{s}=N_{-s} \cdot g \cdot N_{s}=\left(\begin{array}{ll}
a_{s} & b_{s} \\
c_{s} & d_{s}
\end{array}\right)
$$

and $\operatorname{sign} c=c /|c|$ if $c \neq 0$.

Let $0<\tilde{\delta}<\delta$ be so small that if $\Delta(g)<\tilde{\delta}$ then

$$
\Delta\left(g_{s}\right)<1 \text { for all } 0 \leq s \leq 2 D(1) / \delta .
$$

(see (2.5) for the definition of $D(1)$ ). This says that

$$
l=l(g, 1) \geq 2 D(1) / \delta .
$$

Let $\bar{\delta}=\delta / 4 D(1)$ and let $s \in E(g, 1), 0 \leq u \leq \bar{\delta} l$. We have using (2.3) and (2.5)

$$
\begin{gathered}
\left|b_{s+u}\right|=\left|b_{s}\right| \leq D(1) / l^{2} \leq \delta \\
\left|1-a_{s+u}\right|=\left|1-a_{s}-b_{s} u\right| \leq D(1) / l+\bar{\delta} l \cdot D(1) / l^{2} \leq \delta \\
\left|c_{s+u}+c_{s}\right|=\left|b_{s} u^{2}+u\left(a_{s}-d_{s}\right)\right| \leq \bar{\delta}^{2} D(1)+3 \bar{\delta} D(1) \leq 4 D(1) \bar{\delta}=\delta .
\end{gathered}
$$

(2.11) shows that

$$
\left|c_{s+u}\right|>1 \text { if } s+u \notin E(g, 1)
$$

since $\Delta\left(g_{s+u}\right)>1$ for $s+u \notin E(g, 1)$. Also $\left|c_{s}\right| \leq 1$ for $s \in E(g, 1)$. This and (2.12) imply that

$$
\left|c_{s+u}-\operatorname{sign} c_{s+u}\right| \leq \delta \quad \text { if } s+u \notin E(g, 1) .
$$

This completes the proof. 
Denote

$$
W_{\varepsilon}(g)=U(g ; \varepsilon, \varepsilon, 0), g \in G .
$$

We say that $x, y \in G, y \in W_{\varepsilon}(x)$ form an $\varepsilon$-strip of length $t \geq 0$ if for every $s \in[0, t]$ there is $q(s) \geq 0, q(0)=0$ such that

$$
H_{q(s)} y \in W_{\varepsilon}\left(H_{s} x\right) \text {. }
$$

$q(s)=q(s, x, y)$ is uniquely defined by (2.13) and is a smooth function of $(s, x, y)$. It is easy to compute that

$$
|q(s)-s|=D(\varepsilon) s,
$$

where $D(\varepsilon) \rightarrow 0$ when $\varepsilon \rightarrow 0$. It follows from (2.1) that if $x, y$ form an $\varepsilon$-strip of length $t$ then $G_{\tau} x, G_{\tau} y, \tau \geq 0$ form an $\varepsilon$-strip of length $t e^{2 \tau}$.

\section{3. h-invariant partitions}

Let $h=\left\{h_{t}, t \in R\right\}$ be the horocycle flow on $(M=\Gamma \backslash G, \mu)$ and let $S$ on $(Y, \nu)$ be a factor of $h_{1}$ (the time-one transformation of the flow $h_{t}$ ) with a conjugacy $\psi: M \rightarrow Y$

$$
\psi h_{1}(x)=S \psi(x) \text { for a.e. } x \in M
$$

LEMMA 3.1. Let $\zeta$ be the partition of $M$ induced by $\psi$ (see $\S 1$ ). Then there exists $Z \subset M / \zeta, \mu_{\zeta}(Z)>0$ such that $\mu_{C}$ is atomic for every $C \in Z$.

Proof. We can assume without loss of generality that $Y$ is a compact metric space and $S$ is a homeomorphism of $Y$ onto itself. Moreover, there exists $\varepsilon_{Y}>0$ such that

$$
d_{Y}(y, S y)>\varepsilon_{Y} \quad \text { for every } y \in Y,
$$

where $d_{Y}$ denotes the metric in $Y$ (see for instance [3]).

Let $0<\theta<0.01$ be fixed.

Since $\psi: M \rightarrow Y$ is measurable, there is $\Lambda \subset M, \mu(\Lambda)>1-\theta$ such that $\psi$ is uniformly continuous on $\Lambda$ (see lemma 3.1 in [4]).

Let $0<\delta<1$ be such that

$$
\text { if } d\left(w_{1}, w_{2}\right)<\delta, \quad w_{1}, w_{2} \in \Lambda \quad \text { then } d_{Y}\left(\psi w_{1}, \psi w_{2}\right)<\varepsilon_{Y}
$$

Let $\tilde{\delta}=\tilde{\delta}(\delta)>0$ and $\bar{\delta}=\bar{\delta}(\delta)>0$ be as in lemma 2.1. Since $h_{1}$ is ergodic, there are $V \subset M, \mu(V)>1-\bar{\delta} / 100$ and an integer $n_{0}>0$ such that

if $n \geq n_{0}$ and $x \in V$ then the relative frequency of

$\Lambda$ on $\left\{x, h_{1} x, \ldots, h_{n} x\right\}$ is at least $1-2 \theta$.

Let $\tilde{V} \subset M, \mu(\tilde{V})>1-\theta$ and an integer $n_{1}>n_{0}$ be such that

$$
\begin{aligned}
& \text { if } n \geq n_{1} \text { and } x \in \tilde{V} \text { then the relative frequency of } \\
& V \text { on }\left\{x, h_{1} x, \ldots, h_{n} x\right\} \text { is at least } 1-\bar{\delta} / 90 \text {. }
\end{aligned}
$$

Let $0<\delta_{1}<\tilde{\delta}$ be so small that if $d(x, y)<\delta_{1}, x, y \in G$ then

$$
d\left(H_{s} x, H_{s} y\right)<1 \text { for all } 0 \leq s \leq 2 n_{1} / \bar{\delta}
$$


We claim that

$$
d(u, v) \geq \delta_{1}
$$

for every $u, v \in C \cap \tilde{V}, u \neq v$ and every $C \in \zeta$.

Suppose on the contrary that there are $C_{0} \in \zeta$ and $u_{0}, v_{0} \in C_{0} \cap \tilde{V}, u_{0} \neq v_{0}$ such that $d\left(u_{0}, v_{0}\right)<\delta_{1}$.

Let $x_{0}=p^{-1}\left(u_{0}\right), y_{0}=p^{-1}\left(v_{0}\right), x_{0}, y_{0} \in G$ be such that $d\left(x_{0}, y_{0}\right)=d\left(u_{0}, v_{0}\right)$ and let $E=E\left(x_{0}^{-1} \cdot y_{0}, 1\right)=E_{0} \cup E_{1}$ be as in (2.5) ( $E_{1}$ can be empty), $E_{0}=\left[0, s_{0}\right], E_{1}=$ $\left[s_{1}, s_{2}\right], s_{1}>s_{0}$.

(3.5) implies that

$$
2 n_{1} / \bar{\delta} \leq l\left(E_{0}\right) \leq \max \left\{l\left(E_{0}\right), l\left(E_{1}\right)\right\}=l .
$$

\section{Denote}

$$
F_{0}=\left[s_{0}, s_{0}+\bar{\delta} l / 2\right], \quad F=\left[0, s_{0}\right] \cup F_{0} \quad \text { if } s_{1}-s_{0}>\overline{\delta l}
$$

and

$$
F_{0}=\left[s_{2}, s_{2}+\bar{\delta} l / 2\right], \quad F=\left[0, s_{2}\right] \cup F_{0} \quad \text { if } s_{1}-s_{0} \leq \overline{\delta l} .
$$

We have $F_{0} \subset F-E$ and

$$
|F| \geq n_{1} \text { and }\left|F_{0}\right| /|F| \geq \bar{\delta} / 20 .
$$

where $|F|$ denotes the number of integers in $F$.

Let

$$
\tilde{J}=\left\{m \in F: m \text { is an integer and } h_{m} u_{0} \in V, h_{m} v_{0} \in V\right\} .
$$

It follows from (3.4) that

$$
|\tilde{J}| /|F| \geq 1-\bar{\delta} / 40
$$

since $u_{0}, v_{0} \in \tilde{V}$ and $|F|>n_{1}$. This and (3.7) imply that there is an integer $m_{0}$ such that

\section{Denote}

$$
m_{0} \in F_{0} \cap \tilde{J}
$$

$J=\left\{m \in\left[m_{0}, m_{0}+\bar{\delta} l / 2\right]: m\right.$ is an integer and $\left.h_{m} u_{0} \in \Lambda, h_{m-1} v_{0} \in \Lambda, h_{m+1} v_{0} \in \Lambda\right\}$. It follows from (3.3) that

$$
|J| /\left|\left[m_{0}, m_{0}+\bar{\delta} l / 2\right]\right| \geq 1-6 \theta,
$$

since

$$
h_{m_{0}} u_{0}, h_{m_{0}} v_{0} \in V \text { and } \bar{\delta} l / 2>n_{1}>n_{0} .
$$

This implies that there is

$$
m_{1} \in\left[m_{0}, m_{0}+\bar{\delta} l / 2\right] \subset\left[s_{0}, s_{0}+\bar{\delta} l / 2\right] \cup\left[s_{2}, s_{2}+\bar{\delta} l / 2\right]
$$

such that

$$
h_{m_{1}} u_{0} \in \Lambda, \quad h_{m_{1}-1} v_{0} \in \Lambda \text { and } h_{m_{1}+1} v_{0} \in \Lambda .
$$

It follows from lemma 2.1 that

$$
\text { either } d\left(h_{m_{1}} u_{0}, h_{m_{1}+1} v_{0}\right)<\delta \text { or } d\left(h_{m_{1}} u_{0}, h_{m_{1}-1} v_{0}\right)<\delta
$$

since $d\left(u_{0}, v_{0}\right)<\delta_{1}<\tilde{\delta}(\delta)$. 
Assume for simplicity that the first condition of (3.9) holds. We have by (3.8) and our choice of $\delta$

(3.1) implies that

$$
d_{Y}\left(\psi h_{m_{1}} u_{0}, \psi h_{m_{1}+1} v_{0}\right)<\varepsilon_{Y}
$$

Also

$$
\psi\left(h_{m_{1}+1} v_{0}\right)=S \psi\left(h_{m_{1}} v_{0}\right)
$$

$$
\psi\left(h_{m_{1}} u_{0}\right)=\psi\left(h_{m_{1}} v_{0}\right)=y
$$

since $u_{0}, v_{0} \in C_{0} \in \zeta$. (3.10) implies then that

$$
d_{Y}(y, S y)<\varepsilon_{Y}
$$

which contradicts (3.2). So we have proved (3.6).

Since $\mu(\tilde{V})>0$ there is $Z \subset M / \zeta, \mu_{\zeta}(Z)>0$ such that

$$
\mu_{C}(C \cap \tilde{V})>0 \text { for every } C \in Z \text {. }
$$

(3.6) implies that $C \cap \tilde{V}$ is at most countable. This implies via (3.11) that $\mu_{C}$ is atomic for every $C \in Z$. This completes the proof.

Note 3.1. It follows from the proof of lemma 3.1 that given $0<\theta<0.01$ there are a compact $K \subset M, \mu(K)>1-\theta$ and $\delta_{1}>0$ such that

$$
d(u, v) \geq \delta_{1} \quad \text { for every } u, v \in C \cap K, u \neq v \text { and every } C \in \zeta .
$$

\section{Algebraicity of $\xi$}

From now on our discussion will be similar to [4].

Let $S=\left\{S_{i}, t \in R\right\}$ on $(Y, \nu)$ be a factor of $h=\left\{h_{t}, t \in R\right\}$ on $(M, \mu)$ with a conjugacy $\psi: M \rightarrow Y$

$$
\psi h_{t}(x)=S_{t} \psi(x) \text { for all } t \in R \text { and a.e. } x \in M,
$$

and let $\xi$ be the $h$-invariant partition of $M$, induced by $\psi$. It follows from proposition 1.1 and lemma 3.1, that there are $D \subset M, h_{t} D=D, t \in R, \mu(D)=1, U \subset M / \xi$, $h_{t}^{\xi} U=U, t \in R, \mu_{\xi}(U)=1$ and an integer $n>0$ such that for every $C \in U$ the intersection $D \cap C$ consists of exactly $n$ points with $\mu_{C}$-measure $1 / n$.

We assume without loss of generality that $D=M$ and $U=M / \xi$. Thus each $C \in \xi$ consists of $n$ distinct points of $\mu_{C}$-measure $1 / n$.

Let $0<\theta<0.01$ be given. Using the discreteness of $\Gamma \in \mathscr{T}, M=\Gamma \backslash G$ and note 3.1, we can get a compact $K \subset M, \mu(K)>1-\theta^{2} / n^{2}$ and $\rho>0$ such that

(1) if $x \in p^{-1}(K)$ then the projection $p: G \rightarrow M, p(g)=\Gamma g$ is an isometry on the ball of radius $\rho$ centered at $x$.

(2) $d(u, v) \geq \rho$ for every $u, v \in C \cap K, u \neq v, C \in \xi$.

Let

$$
K^{\prime}=\pi^{-1}\left\{C \in M / \xi: \mu_{C}(C \cap K)>1-\frac{\theta}{n}\right\}
$$

where $\pi: M \rightarrow M / \xi$ is the projection $\pi(x)=\xi(x), x \in M$. $K^{\prime}$ consists of atoms of $\xi$. We have

$$
\mu\left(K^{\prime}\right)>1-\theta / n \text { and } \quad K^{\prime} \subset K
$$


since $\mu(K)>1-\theta^{2} / n^{2}$ and every $C \in \xi$ consists of $n$ points of $\mu_{C}$-measure $1 / n$.

Let $0<\varepsilon<\rho / 2$ be so small that

$$
\varepsilon<1 \text { (see }(2.2) \text { ) and } 3 A D(\varepsilon)<\rho / 2 \text { in (2.6). }
$$

Let $0<\delta_{0}<\varepsilon$ be so small that if $d(x, y)<\delta_{0}, x, y \in G$ then

$$
d\left(H_{s} x, H_{s} y\right)<\varepsilon \quad \text { for all } 0 \leq s \leq 1 .
$$

Let $u \in K, v \in M$ and $d(u, v)<\delta<\delta_{0}$. Let $x, y \in G$ be such that $p(x)=u, p(y)=v$ and $d(x, y)<\delta$. Denote

$$
E(u, v, \varepsilon)=E_{0}\left(x^{-1} \cdot y, \varepsilon\right)
$$

where $E_{0}\left(x^{-1} \cdot y, \varepsilon\right)$ is defined in (2.5). $E(u, v, \varepsilon)$ is well defined and does not depend on the choice of $x \in p^{-1}(u), y \in p^{-1}(v)$, since $u \in K$ and $\delta<\rho$. It follows from (4.4) that $l(E(u, v, \varepsilon)) \geq 1$. Henceforth $\xi(v)$ denotes the atom of $\xi$, containing $v$.

LeMMA 4.1. Let $0<\delta<\delta_{0}, u, v \in M$ and $A_{t}=A_{t}(u, v, \delta)=\{s \in[0, t]$ : there exists $v(s) \in \xi(v)$ such that $h_{s} v(s) \in K^{\prime}$ and $\left.d\left(h_{s} u, h_{s} v(s)\right)<\delta\right\}, t \geq 1$. If $l\left(A_{t}\right)>0.9 t$ then there is $s \in A_{t}$ such that $l\left(E\left(h_{s} u, h_{s} v(s), \delta\right)\right) \geq 0.2 t$.

Proof. The proof is similar to that of lemma 2.1 in [4]. Let

$$
E_{s}=s+E\left(h_{s} u, h_{s} v(s), \delta\right), \quad s \in A_{t} .
$$

We claim that

$$
\text { if } s_{1} \in A_{t} \quad \text { and } \quad v\left(s_{1}\right) \neq v(s) \quad \text { then } s_{1} \notin E_{s} \text {. }
$$

Indeed, suppose on the contrary that $s_{1} \in E_{s}$. Then

$$
d\left(h_{s_{1}} u, h_{s_{1}} v(s)\right)<3 A D(\varepsilon)<\rho / 2
$$

by (2.6) and (4.3). Also we have

$$
d\left(h_{s_{1}} v\left(s_{1}\right), h_{s_{1}} u\right)<\delta<\rho / 2,
$$

since $s_{1} \in A_{t}$. This implies that

$$
d\left(h_{s_{1}} v\left(s_{1}\right), h_{s_{1}} v(s)\right)<\rho .
$$

We have

$$
h_{s_{1}} v(s) \in \xi\left(h_{s_{1}} v\left(s_{1}\right)\right)
$$

since $v(s), v\left(s_{1}\right) \in \xi(v)$. Also

$$
h_{s_{1}} v\left(s_{1}\right) \in \boldsymbol{K}^{\prime}
$$

since $s_{1} \in A_{t}$ and therefore

$$
h_{s_{1}} v(s) \in K^{\prime},
$$

since $K^{\prime}$ consists of atoms of $\xi$. This and (4.6) imply that

$$
h_{s_{1}} v(s)=h_{s_{1}} v\left(s_{1}\right)
$$

which contradicts $v(s) \neq v\left(s_{1}\right)$ in $(4.5)$. 
Let $\beta=\left\{E_{1}, \ldots, E_{m}\right\}$ be the collection of pairwise disjoint intervals $E_{i}=$ $\left[s_{i}, \tau_{i}\right] \subset[0, t], s_{j}>\tau_{i}, j>i$, such that $E_{i}=E_{s}$ for some $s \in A_{t}, i=1, \ldots, m$ and $A_{t} \subset \bigcup_{i=1}^{m} E_{i}$ and let $d\left(E_{i}, E_{j}\right)=s_{j}-\tau_{i}$.

Let $x \in G$ be such that $p(x)=u, x_{i}=H_{s_{i}} x, p\left(x_{i}\right)=h_{s_{i}} u=u_{i}$ and let $y_{i} \in G$ be such that $d\left(x_{i}, y_{i}\right)<\delta$ and $p\left(y_{i}\right)=h_{s_{i}} v\left(s_{i}\right)=v_{i}$. We have

$$
E_{i}=s_{i}+E_{0}\left(x_{i}^{-1} \cdot y_{i}, \delta\right) \subset s_{i}+E\left(x_{i}^{-1} \cdot y_{i}, \delta\right)
$$

and

$$
l\left(E_{i}\right) \leq l\left(x_{i}^{-1} \cdot y_{i}, \delta\right)=l_{i}
$$

(see (2.5)). Suppose that $s_{j}-s_{i}=q$ and $v\left(s_{i}\right)=v\left(s_{j}\right)$. We have

$$
\left(h_{s_{i}} u, h_{s_{i}} v\left(s_{j}\right)\right)=\left(u_{j}, v_{i}\right)=\left(h_{q} u_{i}, h_{q} v_{i}\right) .
$$

Though $d\left(x_{i}, y_{i}\right)<\delta, p\left(x_{i}\right)=u_{i}, p\left(y_{i}\right)=v_{i}$ and $d\left(u_{j}, v_{j}\right)<\delta$, it is not necessarily true that

$$
d\left(H_{q} x_{i}, H_{q} y_{i}\right)<\delta,
$$

but there is a unique $\mathscr{D} \in \Gamma$ such that

$$
d\left(H_{q} x_{i}, \mathscr{D} \cdot H_{q} y_{i}\right)<\delta .
$$

We write $E_{i} \stackrel{\Gamma}{\sim} E_{j}$ if $v\left(s_{i}\right)=v\left(s_{j}\right)$ and $\mathscr{D} \neq e$ in (4.7), $E_{i} \sim E_{j}$ if $v\left(s_{i}\right)=v\left(s_{j}\right)$ and $\mathscr{D}=e$ in (4.7) and $E_{i} \stackrel{\xi}{\xi} E_{j}$ if $v\left(s_{i}\right) \neq v\left(s_{j}\right)$. It follows from (2.6) and (4.3) that

$$
d\left(H_{q_{i}+s} x_{i}, H_{q_{i}+s} y_{i}\right) \leq 3 A D(\varepsilon)<\rho / 2
$$

for all $0 \leq s \leq l_{i}$, where $q_{i}=\tau_{i}-s_{i}, i=1, \ldots, m$. This implies via (4.1) that

$$
s_{j}-\tau_{i}=d\left(E_{i}, E_{j}\right) \geq l_{i} \text { if } E_{i} \stackrel{\Gamma}{\sim} E_{j}
$$

since $y_{j} \in p^{-1}(K)$. (4.8) also shows that

$$
d\left(h_{\tau_{i}+s} u, h_{\tau_{i}+s} v\left(s_{i}\right)\right)=d\left(h_{q_{i}+s} u_{i}, h_{q_{i}+s} v_{i}\right)<\rho / 2
$$

for all $0 \leq s \leq l_{i}$. This implies that

$$
s_{j}-\tau_{i}=d\left(E_{i}, E_{j}\right) \geq l_{i} \quad \text { if } \stackrel{\stackrel{\xi}{\sim}}{\sim} E_{j},
$$

since otherwise we would have

$$
d\left(h_{s_{i}} v\left(s_{i}\right), h_{s_{j}} v\left(s_{j}\right)\right)<\rho
$$

which contradicts $(4.1)$, since $v\left(s_{i}\right) \neq v\left(s_{j}\right), h_{s_{j}} v\left(s_{j}\right) \in K^{\prime}$ and $h_{s_{j}} v\left(s_{i}\right) \in \xi\left(h_{s_{j}} v\left(s_{j}\right)\right) \subset K^{\prime}$.

Let us now define a new collection $\bar{\beta}=\left\{\bar{E}_{1}, \ldots, \bar{E}_{\bar{m}}\right\}$ by the following procedure. We set $\bar{E}_{1}=E_{1}$ unless $E_{1} \stackrel{\mathscr{\sim}}{\sim} E_{2}$ and $d\left(E_{1}, E_{2}\right) \leq l\left(E_{1}\right)$. In this last case we set $\bar{E}_{1}=\left[s_{1}, \tau_{2}\right] \supset E_{1} \cup E_{2}$. Suppose $\bar{E}_{k}, k=1, \ldots, p$ have been defined. To define $\bar{E}_{p+1}$ we apply the same construction to the first $E \in \beta$, which has not been included in any $\bar{E}_{k}, k=1, \ldots, p$.

It follows from the construction of $\bar{\beta}$ that

$$
d\left(\bar{E}_{k}, \bar{E}_{k+1}\right) \geq l\left(\bar{E}_{k}\right) \quad \text { if } \bar{E}_{k} \stackrel{e}{\sim} \bar{E}_{k+1}
$$


and for each $\vec{E}_{k} \in \vec{\beta}$ there is $E_{i_{k}} \in \beta$ such that

$$
\text { either } \bar{E}_{k}=E_{i_{k}} \quad \text { or } \quad \bar{E}_{k} \supset\left(E_{i_{k}} \cup E_{i_{k}+1}\right) \text { and } l\left(\bar{E}_{k}\right) \leq 3 l_{i_{k}} \text {. }
$$

This, (4.9) and (4.10) imply

$$
d\left(\bar{E}_{k}, \bar{E}_{k+1}\right) \geq l_{i_{k}} \geq l\left(\bar{E}_{k}\right) / 3
$$

if $\bar{E}_{k} \stackrel{\Gamma}{\sim} \bar{E}_{k+1}$ or $\bar{E}_{k} \stackrel{\xi}{\sim} \bar{E}_{k+1}$. This and (4.11) give

Denote

$$
d\left(\bar{E}_{k}, \bar{E}_{k+1}\right) \geq l\left(\bar{E}_{k}\right) / 3 \quad \text { for all } k=1, \ldots, \bar{m}-1 .
$$

$$
l(\bar{\beta})=\sum_{k=1}^{\tilde{m}} l\left(\bar{E}_{k}\right)
$$

We have

$$
l(\bar{\beta})>0.9 t,
$$

since $A_{t} \subset \bigcup_{k=1}^{m} \bar{E}_{k}$.

This and (4.13) imply that there is $\bar{E} \in \bar{\beta}$ such that

$$
l(\bar{E}) \geq 0.6 t \text {. }
$$

This implies via (4.12) that there is $E \in \beta$ such that $l(E) \geq 0.2 t$. This completes the proof.

Corollary 4.1. Let $u, v \in M$ and let $l\left(A_{t}\right)>0.9 t$ for all $t \geq t_{0}>1$, where $A_{t}=$ $A_{t}(u, v, \delta)$ as in lemma 4.1. Then there is $\tilde{v} \in \xi(v)$ such that $\tilde{v}=h_{q} u$ for some $q=q(u, v, \delta),|q|<\delta$.

Proof. It follows from the proof of lemma 4.1 that there is $s \geq 0$ such that

$$
l\left(E\left(h_{s} u, h_{s} v(s), \delta\right)\right) \geq 0.2 t \text { for all } t \geq t_{0} .
$$

(2.5) shows that this may happen only if $h_{s} v(s)=h_{q} h_{s} u$ for some $|q|<\delta$. We get $\tilde{v}=v(s)=h_{q} u, \tilde{v} \in \xi(v)$.

For $A \subset M$ we shall write $A<\xi$ if $A$ consists of atoms of $\xi$.

According to $\S 1$ there are $X<\xi, \mu(X)=1$ and pairwise disjoint measurable sets

$$
X_{i} \subset X, i=1, \ldots n, \bigcup_{i=1}^{n} X_{i}=X, \mu\left(X_{i}\right)=\frac{1}{n}
$$

such that for every $x \in X$ the intersection

$$
\xi(x) \cap X_{i}=\left\{x_{i}(x)\right\}
$$

consists of exactly one point and the map $\phi_{i}: X$ onto $X_{i}$ defined by $\phi_{i}(x)=x_{i}(x)$ is measurable, $i=1, \ldots, n$.

Let $K^{\prime}$ be the set defined in (4.2) and let

$$
\tilde{K}=K^{\prime} \cap X, \quad \mu(\tilde{K})=\mu\left(\tilde{K}^{\prime}\right)>1-\frac{\theta}{n}, \quad \tilde{K}<\xi .
$$


Since $\phi_{i}: X \rightarrow X_{i}$ is measurable, $i=1, \ldots, n$ there is $\Lambda \subset X, \mu(\Lambda)>1-\theta$ such that $\Lambda<\xi$ and each $\phi_{i}, i=1, \ldots, n$ is uniformly continuous on $\Lambda$ (see lemma 3.1 in [4]).

Let

$$
Q=\Lambda \cap \tilde{K}, \quad \mu(Q)>1-2 \theta, \quad Q<\xi
$$

and let $\Omega$ be the generic set of $Q$ for $h$,

$$
h_{t} \Omega=\Omega, \quad t \in R, \quad \mu(\Omega)=1, \quad \Omega<\xi .
$$

LEMMA 4.2. For every $0<\delta<\delta_{0}$ there is $\omega=\omega(\delta)>0$ such that if $u_{1}, v_{1} \in \Omega, v_{1}=g_{p} u_{1}$ for some $|p|<\omega$, then for every $u_{2} \in \xi\left(u_{1}\right)$ there is $v_{2} \in \xi\left(v_{1}\right)$ such that $v_{2}=h_{b} g_{p} u_{2}$ for some $b=b\left(u_{1}, u_{2}, p\right),|b|<\delta$ and $b\left(h_{1} u_{1}, h_{t} u_{2}, p\right)=b\left(u_{1}, u_{2}, p\right)$ for all $t \in R$.

Proof. Since $\phi_{i}, i=1, \ldots, n$ are uniformly continuous on $\Lambda$ there is $0<\omega<\delta / 2$ such that

if $d\left(w_{1}, w_{2}\right)<\omega, w_{1}, w_{2} \in \Lambda$ then $d\left(\phi_{i} \cdot w_{1}, \phi_{i} \cdot w_{2}\right)<\delta / 2, i=1, \ldots, n$.

Let $u_{1}, v_{1} \in \Omega, v_{1}=g_{p} u_{1}$ for some $|p|<\omega$. Let $\lambda_{0}>0$ be such that

$$
\begin{aligned}
& \text { if } \lambda \geq \lambda_{0} \text { then the relative length measure of } Q \text { on } \\
& {\left[u_{1}, h_{\lambda} u_{1}\right] \text { and on }\left[v_{1}, h_{\lambda} v_{1}\right] \text { is at least } 1-3 \theta .}
\end{aligned}
$$

Let $x, y \in G, y=G_{p} x$ be such that $p(x)=u_{1}, p(y)=v_{1} . x$ and $y$ form an $\omega$-strip of length $\lambda$ for every $\lambda>0$. We have

$$
H_{q(s)} y=G_{p} H_{s} \quad(\text { see }(2.13)) \text { and } \quad h_{q(s)} v_{1}=g_{p} h_{s} u_{1} \quad \text { for all } s \geq 0 .
$$

Denote

$$
F_{\lambda}=\left\{s \in[0, \lambda]: h_{s} u_{1} \in Q, h_{q(s)} v_{1} \in Q\right\} .
$$

It follows from $(4.15)$ that

$$
l\left(F_{\lambda}\right)>(1-7 \theta) \lambda
$$

if $\omega>0$ is sufficiently small and $\lambda \geq \lambda_{0}, q(\lambda) \geq \lambda_{0}$ (see (2.14)).

Let $u_{2} \in \xi\left(u_{1}\right)$. We write $j(t)=i \in\{1, \ldots, n\} \quad$ if $h_{t} u_{2} \in X_{i}$.

We have

$$
\begin{gathered}
\phi_{j(s)}\left(h_{s} u_{1}\right)=h_{s} u_{2} \in X_{j(s)} \\
\phi_{j(s)}\left(h_{q(s)} v_{1}\right) \in \xi\left(h_{q(s)} v_{1}\right)=h_{q(s)} \xi\left(v_{1}\right)
\end{gathered}
$$

or

$$
\phi_{j(s)}\left(h_{q(s)} v_{1}\right)=h_{q(s)} v_{1}(q(s)),
$$

where $v_{1}(q(s)) \in \xi\left(v_{1}\right)$ and if $s \in F_{\lambda}$ then

and

$$
\begin{gathered}
h_{s} u_{2} \in K^{\prime}, \quad h_{q(s)} v_{1}(q(s)) \in K^{\prime} \\
d\left(h_{s} u_{2}, h_{q(s)} v_{1}(q(s))\right)<\delta / 2
\end{gathered}
$$

by (4.14). Let $w=g_{p} u_{2}$. We have

$$
h_{q(s)} w=g_{p} h_{s} u_{2}
$$

and therefore

$$
d\left(h_{s} u_{2}, h_{q(s)} w\right)<\omega
$$


This and (4.17) imply that

$$
d\left(h_{q(s)} w, h_{q(s)} v_{1}(q(s))\right)<\omega+\delta / 2 \leq \delta
$$

for all $s \in F_{\lambda}$ and all $\lambda \geq \lambda_{0}, q(\lambda) \geq \lambda_{0}$.

Let $A_{t}=A_{t}\left(w, v_{1}, \delta\right)$ be as in lemma 4.1. (4.16) and (4.18) show that there is $t_{0}>1$ such that

$$
l\left(A_{t}\right)>0.9 t \text { for all } t \geq t_{0} \text {. }
$$

It follows then from corollary 4.1 that there is $v_{2} \in \xi\left(v_{1}\right)$ such that $v_{2}=h_{b} w=h_{b} q_{p} u_{2}$ for some $b=b\left(u_{1}, u_{2}, p\right),|b|<\delta$. It is clear, that $b\left(h_{t} u_{1}, h_{t} u_{2}, p\right)=b\left(u_{1}, u_{2}, p\right)$ for all $t \in R,|p|<\omega$.

It follows from lemma 4.2 that there exists $\omega_{0}>0$ such that

$$
g_{p} w \in \Omega \text { iff } g_{p} u \in \Omega
$$

for every $u \in \Omega, w \in \xi(u),|p|<\omega_{0}$, since $\Omega$ is $h$-invariant and $\Omega<\xi$.

Let

$$
\Omega_{p}=\left\{u \in \Omega: g_{p} u \in \Omega\right\},|p|<\omega_{0} .
$$

$\Omega_{p}$ is $h$-invariant, $\mu\left(\Omega_{p}\right)=1$ and $\Omega_{p}<\xi$.

LEMMA 4.3. There is an $h$-invariant $\Omega_{p}^{\prime} \subset \Omega_{p}, \Omega_{p}^{\prime}<\xi, \mu\left(\Omega_{p}^{\prime}\right)=1$ such that $b(u, w, p)=0$ for all $u \in \Omega_{p}^{\prime}, w \in \xi(u),|p|<\omega_{0}$.

Proof. It follows from the definition of $b(u, w, p)$ that it is measurable and

$$
\begin{gathered}
b(u, w, p)=-b(w, u, p) \\
b(x, w, p)=b(u, w, p)-b(u, x, p), x, w \in \xi(u), \\
u \in \Omega_{p},|p|<\omega_{0} .
\end{gathered}
$$

Define $\bar{f}_{p}: \Omega_{p} \rightarrow R$ and $\tilde{f}_{p}: \Omega_{p} \rightarrow R$ by

$$
\begin{aligned}
& \bar{f}_{p}(u)=\max \{b(u, w, p): w \in \xi(u)\} \\
& \tilde{f}_{p}(u)=\min \{b(u, w, p): w \in \xi(u)\} .
\end{aligned}
$$

The functions $\bar{f}_{p}$ and $\tilde{f}_{p}$ are measurable and constant on orbits of $h$. Since $h$ is ergodic, there are $\Omega_{p}^{\prime} \subset \Omega_{p}, \mu\left(\Omega_{p}^{\prime}\right)=1, \Omega_{p}^{\prime}<\xi$ and constants $\bar{\sigma}, \tilde{\sigma}$ such that $\bar{f}_{p}=\bar{\sigma}$ and $\tilde{f}_{p}=\tilde{\sigma}$ on $\Omega_{p}^{\prime}$.

We claim that $\bar{\sigma}=\tilde{\sigma}=0$. Indeed, suppose on the contrary that $\bar{\sigma}>0$. Let $u \in \Omega_{p}^{\prime}$ and $w \in \xi(u)$ be such that

Then

$$
b(u, w, p)=\bar{\sigma}
$$

$$
b(w, u, p)=-\bar{\sigma}<0
$$

and therefore $\tilde{\sigma}<0$.

Let $x \in \xi(u)$ be such that

$$
b(u, x, p)=\tilde{\sigma} .
$$

Then

$$
b(x, w, p)=\bar{\sigma}-\tilde{\sigma}>\bar{\sigma}
$$


by (4.19) which contradicts the fact that $\bar{\sigma}=\max \{b(x, w, p): w \in \xi(x)\}$. Therefore $\bar{\sigma}=\tilde{\sigma}=0$. This completes the proof.

Let

$$
\tilde{\Omega}=\bigcap_{\substack{p \text { is rational } \\|p|<\omega_{0}}} \Omega_{p}^{\prime}
$$

$\tilde{\Omega}$ is $h$-invariant, $\mu(\tilde{\Omega})=1$ and $\tilde{\Omega}<\xi$. We have

$$
g_{p}(\xi(u))=\xi\left(g_{p} u\right)
$$

for all $u \in \tilde{\Omega}$ and all rational $|p|<\omega_{0}$.

Let $\bar{\Omega}=\{u \in M: \tilde{\Omega}$ is dense on the geodesic orbit of $u\} . \bar{\Omega}$ is $h$-invariant, $\mu(\bar{\Omega})=1$ and $\bar{\Omega} \cap \tilde{\Omega}<\xi$. Lemma 4.2 shows that $b(u, w, p)$ is continuous in $p$. This implies that

$$
g_{p}(\xi(u))=\xi\left(g_{p} u\right)
$$

for all $u \in \bar{\Omega} \cap \tilde{\Omega}$ and all $p \in R$ with $g_{p} u \in \Omega$.

Let $g_{p} u \in M-\Omega$ for some $u \in \bar{\Omega} \cap \tilde{\Omega}, p \in R$. We have

$$
\begin{gathered}
\xi\left(g_{p} u\right) \subset M-\Omega, \quad \text { since } \Omega<\xi ; \\
g_{p}(\xi(u)) \subset M-\Omega \quad \text { by }(4.20) .
\end{gathered}
$$

Let us define a partition $\bar{\xi}$ on $\bar{\Omega}$ by

$$
\begin{aligned}
& \bar{\xi}\left(g_{p} u\right)=\xi\left(g_{p} u\right) \quad \text { if } u \in \bar{\Omega} \cap \tilde{\Omega}, g_{p} u \in \Omega \\
& \bar{\xi}\left(g_{p} u\right)=g_{p}(\xi(u)) \quad \text { if } u \in \bar{\Omega} \cap \tilde{\Omega}, g_{p} u \notin \Omega .
\end{aligned}
$$

We have

$$
\bar{\xi}=\xi \text { on } \bar{\Omega} \cap \Omega<\xi \quad h_{t} \bar{\xi}(u)=\bar{\xi}\left(h_{t} u\right) \quad g_{t} \bar{\xi}(u)=\bar{\xi}\left(g_{t} u\right)
$$

for all $u \in \bar{\Omega}$ and all $t \in R$.

Let $Q \subset M, \mu(Q)>1-2 \theta, Q<\xi$ be as in lemma 4.2. Since $h$ is ergodic, there are $Z \subset \Omega, Z<\xi, \mu(Z)>1-\theta$ and $i>0$ such that

$$
\begin{aligned}
& \text { if } z \in Z, t>i \text { then the relative length measure of } Q \\
& \text { on }\left[z, h_{t} z\right] \text { is at least } 1-3 \theta \text {. }
\end{aligned}
$$

Let $\bar{Z} \subset \bar{\Omega}$ be the generic set of $Z$ for the geodesic flow $g, \bar{Z}<\bar{\xi}, \mu(\bar{Z})=1$.

LEMMA 4.4. There exists $\gamma>0$ such that if $u, v \in \bar{Z}$ and $v=h_{r}^{*} u$ for some $|r|<\gamma$ then

$$
\bar{\xi}(v)=h_{r}^{*} \bar{\xi}(u) \text {. }
$$

Proof. The proof is similar to that of lemma 4.2. Since $\phi_{i}, i=1, \ldots, n$ are uniformly continuous on $Q$, given $0<\delta<\delta_{0}$ there is $0<\omega=\omega(\delta)<\delta / 2$ such that

if $d\left(w_{1}, w_{2}\right)<\omega, \quad w_{1}, w_{2} \in Q$ then $d\left(\phi_{i} w_{1}, \phi_{i} w_{2}\right)<\delta / 2$ for all $i=1, \ldots, n$.

Let $0<\gamma<\omega$ be such that if $x, y \in G, y \in W_{\gamma}(x)$ then $x, y$ form an $\omega$-strip of length 1 (see (2.13)). Let

$$
u, v \in \bar{Z}, v=h_{r}^{*} u \text { for some }|r|<\gamma
$$


We shall show that

$$
h_{r}^{*} u_{1} \in \bar{\xi}(v) \text { for every } u_{1} \in \bar{\xi}(u) .
$$

Let $x, y \in G, p(x)=u, p(y)=v, y=H_{r}^{*} x . x$ and $y$ form an $\omega$-strip of length 1 .

Since $u, v \in \bar{Z}$, there is a sequence $0<\tau_{k} \rightarrow \infty, k \rightarrow \infty$ such that $\exp \left(2 \tau_{k}\right)>i$ and

$$
u^{(k)}=g_{\tau_{k}} u \in Z, \quad v^{(k)}=g_{\tau_{k}} v \in Z, k=1,2, \ldots
$$

Let $x^{(k)}=G_{\tau_{k}} x, y^{(k)}=G_{\tau_{k}} y$. We have $p\left(x^{(k)}\right)=u^{(k)}, p\left(y^{(k)}\right)=v^{(k)}$ and $x^{(k)}, y^{(k)}$ form an $\omega$-strip of length $t_{k}=\exp \left(2 \tau_{k}\right)>\bar{t}$. This means (see (2.13)) that

$$
H_{q(s)} y^{(k)} \in W_{\omega}\left(H_{s} x^{(k)}\right) \text { for all } s \in\left[0, t_{k}\right]
$$

or

$$
h_{q(s)} v^{(k)} \in W_{\omega}\left(h_{s} u^{(k)}\right), \quad s \in\left[0, t_{k}\right]
$$

Let

$$
B_{k}=\left\{s \in\left[0, t_{k}\right]: h_{s} u^{(k)} \in Q, h_{q(s)} v^{(k)} \in Q\right\} .
$$

$k=1,2, \ldots(4.22)$ implies that

$$
l\left(B_{k}\right)>(1-7 \theta) t_{k}, \quad k=1,2, \ldots
$$

if $\omega$ is sufficiently small, $t_{k}>\bar{i}, q\left(t_{k}\right)>\bar{i}$.

Let $u_{1} \in \bar{\xi}(u)$. Then

$$
u_{1}^{(k)}=g_{\tau_{k}} u_{1} \in \bar{\xi}\left(u^{(k)}\right)
$$

by (4.21). We write $j_{k}(s)=i \in\{1, \ldots, n\}$ if $h_{s} u_{1}^{(k)} \in X_{i}$. We have that if $s \in B_{k}$ then

$$
\begin{gathered}
h_{s} u_{1}^{(k)}=\phi_{j_{k}(s)} h_{s} u^{(k)} \\
\phi_{j_{k}(s)} h_{q(s)} v^{(k)} \in \xi\left(h_{q(s)} v^{(k)}\right)=h_{q(s)}\left(\xi\left(v^{(k)}\right)\right)
\end{gathered}
$$

or

$$
\phi_{j_{k}(s)} h_{q(s)} v^{(k)}=h_{q(s)} v^{(k)}(q(s))
$$

for some

since $v^{(k)} \in Z \subset \Omega$, and

$$
v^{(k)}(q(s)) \in \xi\left(v^{(k)}\right)=\bar{\xi}\left(v^{(k)}\right),
$$

$$
d\left(h_{s} u_{1}^{(k)}, h_{q(s)} v^{(k)}(q(s))\right)<\delta / 2 \quad k=1,2, \ldots
$$

by (4.23). Let

$$
w=h_{r}^{*} u_{1} \quad \text { and } \quad w^{(k)}=g_{\tau_{k}} w, \quad k=1,2, \ldots
$$

We have

$$
d\left(h_{s} u_{1}^{(k)}, h_{q(s)} w^{(k)}\right)<\omega, \quad s \in\left[0, t_{k}\right], \quad k=1,2, \ldots
$$

This and (4.25) imply that

$$
d\left(h_{q(s)} w^{(k)}, h_{q(s)} v^{(k)}(q(s))\right)<\omega+\delta / 2<\delta .
$$

Also

$$
h_{q(s)} v^{(k)}(q(s)) \in K^{\prime} \quad \text { if } s \in B_{k}
$$


Let

$$
A_{k}=A_{q\left(t_{k}\right)}\left(w^{(k)}, v^{(k)}, \delta\right) \subset\left[0, q\left(t_{k}\right)\right]
$$

be as in lemma 4.1. We have

$$
l\left(A_{k}\right) \geq 0.9 q\left(t_{k}\right), \quad k=1,2, \ldots
$$

by (4.24) and (4.26), if $\omega$ is sufficiently small. This implies via lemma 4.1 that there is $s_{k} \in\left[0, q\left(t_{k}\right)\right]$ such that

$$
E\left(h_{s_{k}} w^{(k)}, h_{s_{k}} v^{(k)}\left(s_{k}\right), \delta\right) \geq 0.2 q\left(t_{k}\right), \quad k=1,2, \ldots
$$

This implies via (2.9) that

$$
h_{s_{k}} v^{(k)}\left(s_{k}\right) \in U\left(h_{s_{k}} w^{(k)}, D(\varepsilon) / t_{k}, D(\varepsilon) / t_{k}^{2}, D(\varepsilon)\right)
$$

and therefore

$$
\begin{aligned}
g_{-\tau_{k}} h_{s_{k}} v^{(k)}\left(s_{k}\right) & =h_{s_{k} \exp \left(-2 \tau_{k}\right)} g_{-\tau_{k}} v^{(k)}\left(s_{k}\right) \\
& =h_{s_{k} \exp \left(-2 \tau_{k}\right)} \tilde{v}(k) \in U\left(h_{s_{k} \exp \left(-2 \tau_{k}\right)} w, D(\varepsilon) / t_{k}, D(\varepsilon) / t_{k}, D(\varepsilon) / t_{k}\right), \\
& k=1,2, \ldots
\end{aligned}
$$

where $\tilde{v}(k)=g_{-\tau_{k}} v^{(k)}\left(s_{k}\right) \in \bar{\xi}(v)$ by (4.21). (4.27) may happen only if

$$
w=h_{r}^{*} u_{1} \in \bar{\xi}(v)
$$

since $s_{k} \exp \left(-2 \tau_{k}\right) \in[0, q(1)], k=1,2, \ldots$, and $\bar{\xi}(v)$ is finite. This completes the proof.

For $w \in M$ we denote

$$
W^{(u)}(w)=\left\{w^{\prime} \in M: w^{\prime}=h_{r} g_{p} w \text { for some } p, r \in R\right\} .
$$

$W^{(u)}(w), w \in M$ form the unstable foliation $W^{(u)}$ for the geodesic flow $g$. The set $\bar{\Omega}$ consists of leaves of $W^{(u)}$. It follows from (4.21) that if $w_{k} \in W^{(u)}(w), w \in \bar{\Omega}$ and $w_{k} \rightarrow w$ in the topology of $W^{(u)}(w)$, then

$$
\bar{\xi}\left(w_{k}\right) \rightarrow \bar{\xi}(w), \quad k \rightarrow \infty .
$$

Let

$$
\tilde{Z}=\left\{w \in \bar{\Omega}: \bar{Z} \text { is dense on the } h^{*} \text {-orbit of } w\right\},
$$

$\mu(\tilde{Z})=1$ and let

$$
\bar{W}=\left\{w \in \bar{\Omega}: \bar{Z} \cap \tilde{Z} \text { is dense in } W^{(u)}(w)\right\}, \mu(\bar{W})=1 .
$$

It follows from lemma 4.4 and (4.21) that $\bar{W}<\bar{\xi}$ and

if $u, v \in \bar{W}, v=h_{q} h_{r}^{*} g_{p} u \quad$ for some $p, q, r \in R$ then $\bar{\xi}(v)=h_{q} h_{r}^{*} q_{p} \bar{\xi}(u)$.

This implies that if

$$
w_{k} \in \bar{W}, \quad w_{k}^{\prime} \in \bar{W}, \quad w_{k} \rightarrow w \in M, \quad w_{k}^{\prime} \rightarrow w
$$

when $k \rightarrow \infty$ then

$$
\lim _{k \rightarrow \infty} \bar{\xi}\left(w_{k}\right)=\lim _{k \rightarrow \infty} \bar{\xi}\left(w_{k}^{\prime}\right)
$$

and this limit equals $\bar{\xi}(w)$, if $w \in \bar{W}$. This implies that

$$
\text { if } w \in M-\bar{W}, \quad w_{k} \rightarrow w, \quad w_{k} \in \bar{W} \quad \text { then } \lim _{k \rightarrow \infty} \bar{\xi}\left(w_{k}\right) \subset M-\bar{W} .
$$


Let us define a partition $\bar{\xi}$ on $M$ by

$$
\begin{aligned}
& \tilde{\xi}(u)=\bar{\xi}(u) \quad \text { if } u \in \vec{W} \text { and } \\
& \tilde{\xi}(u)=\lim _{k \rightarrow \infty} \bar{\xi}\left(u_{k}\right), u_{k} \in \bar{W}, u_{k} \rightarrow u, k \rightarrow \infty
\end{aligned}
$$

$\tilde{\xi}$ is well defined and

$$
\begin{aligned}
& \tilde{\xi}=\xi \text { on } \bar{W} \cap \bar{\Omega} \cap \Omega \text { by (4.21) and if } v=h_{q} h_{r}^{*} g_{p} u, u \in M \\
& \text { then } \tilde{\xi}(v)=h_{q} h_{r}^{*} g_{p} \tilde{\xi}(u) \text { by (4.28). }
\end{aligned}
$$

(4.29) shows that $h^{\xi}$ on $M / \xi$ and $h^{\bar{\xi}}$ on $M / \tilde{\xi}$ are isomorphic, since $\bar{W} \cap \bar{\Omega} \cap \Omega$ is $h$-invariant and $\mu(\bar{W} \cap \bar{\Omega} \cap \Omega)=1$.

Proof of theorem 1. Denote

$$
\tilde{\Gamma}(u)=p^{-1}(\tilde{\xi}(u)), u \in M \quad \text { and } \quad \tilde{\Gamma}=\tilde{\Gamma}\left(u_{o}\right),
$$

where $u_{0}=p(e)$. We shall show that $\tilde{\Gamma}$ is a subgroup of $G$.

We say that $J \in G$ is a chain in $G$ if $J=J_{1} \cdots J_{k}$ where

$$
J_{i}=H_{q_{i}} H_{r_{i}}^{*} G_{p_{i}} e=e \cdot\left(\begin{array}{ll}
\exp \left(p_{i}\right) & \exp \left(-p_{i}\right)
\end{array}\right) \cdot\left(\begin{array}{ll}
1 & r_{i} \\
0 & 1
\end{array}\right) \cdot\left(\begin{array}{cc}
1 & 0 \\
q_{i} & 1
\end{array}\right)
$$

for some $p_{i}, q_{i}, r_{i} \in R, i=1, \cdots, k$. It is clear, that for any $g_{1}, g_{2} \in G$ there is a chain $J \in G$ such that $g_{2}=g_{1} \cdot J$.

Let $g, \tilde{g} \in \tilde{\Gamma}$ and let

$$
g=e \cdot J, \quad \tilde{g}=e \cdot \tilde{J}
$$

for some chains

$$
J=J_{1} \cdots J_{k}, \quad J_{i}=H_{q_{i}} H_{r_{i}}^{*} G_{p_{i}} e, \quad i=1, \ldots, k
$$

and

$$
\tilde{J}=\tilde{J}_{1} \ldots \tilde{J}_{k}, \quad \tilde{J}_{i}=H_{\tilde{q}_{i}} H_{\tilde{f}_{i}}^{*} G_{\tilde{p}_{i}} e, \quad i=1, \ldots, \tilde{k}
$$

We write

$$
p\left(J_{i}\right)=h_{q_{i}} h_{r_{i}}^{*} g_{p_{i}} p(e)=\left(h h^{*} g\right)_{i}\left(u_{0}\right), \quad i=1, \ldots, k
$$

We have

$$
\begin{aligned}
& p(g)=\left(h h^{*} g\right)_{k} \cdots\left(h h^{*} g\right)_{1}\left(u_{0}\right) \in \tilde{\xi}\left(u_{0}\right) \\
& p(\tilde{g})=\left(\widetilde{h h^{*} g}\right)_{k} \cdots\left(\widetilde{h h^{*} g}\right)_{1}\left(u_{0}\right) \in \tilde{\xi}\left(u_{0}\right)
\end{aligned}
$$

since $g, \tilde{g} \in \tilde{\Gamma}$. This implies by (4.29) that

$$
\left(h h^{*} g\right)_{k} \cdots\left(h h^{*} g\right)_{1}\left(\tilde{\xi}\left(u_{0}\right)\right)=\tilde{\xi}\left(u_{0}\right)
$$

and

$$
\left(\widetilde{h h^{*} g}\right)_{\tilde{k}} \cdots\left(\widetilde{h h^{*} g}\right)_{1}\left(\tilde{\xi}\left(u_{0}\right)\right)=\tilde{\xi}\left(u_{0}\right)
$$

We have

and

$$
g \cdot \tilde{g}=e \cdot J \cdot \tilde{J}
$$

$$
p(g \cdot \tilde{g})=\left(\widetilde{h h^{*} g}\right)_{\tilde{k}} \cdots\left(\widetilde{h h^{*} g}\right)_{1}\left(h h^{*} g\right)_{k} \cdots\left(h h^{*} g\right)_{1}\left(u_{0}\right) \in \tilde{\xi}\left(u_{0}\right)
$$

by $(4.30)$. 
This implies that $g \cdot \tilde{g} \in \tilde{\Gamma}$ and that $\tilde{\Gamma}$ is a subgroup of $G$. It is clear that $\tilde{\Gamma}$ is discrete and $\Gamma \subset \tilde{\Gamma}$.

Let $g \in \tilde{\Gamma}(u), u \in M$ and let $g=e \cdot J$ for some chain $J \in G$. (4.29) shows that then

$$
\tilde{\Gamma}(u)=\tilde{\Gamma} \cdot J=\tilde{\Gamma} g .
$$

Define $\tilde{\psi}: \tilde{\Gamma} / G$ onto $M / \tilde{\xi}$ by

$$
\tilde{\psi}(\tilde{\Gamma} g)=\tilde{\xi}(p(g))
$$

It is clear that $\tilde{\psi}$ is measure preserving and

$$
\tilde{\psi} \tilde{h}_{t}(\tilde{\Gamma} g)=\tilde{\psi}\left(\tilde{\Gamma} g \cdot N_{t}\right)=\tilde{\xi}\left(p\left(g \cdot N_{t}\right)\right)=\tilde{\xi}\left(h_{t} g\right)=h_{t}^{\tilde{\xi}} \tilde{\xi}(g) .
$$

This shows that $\tilde{\psi}$ is an isomorphism between $\tilde{h}$ and $\tilde{\Gamma} / G$ and $h^{\tilde{\xi}}$ on $M / \tilde{\xi}$. This implies via (4.29) that $\tilde{h}$ is isomorphic to $h^{\xi}$ on $M / \xi$.

\section{Proof of theorem 3}

Let $S$ on $(Y, \nu)$ be a factor of $h_{1}$ on $(M=\Gamma \backslash G, \mu)$ with a conjugacy $\psi: M \rightarrow Y$

$$
\psi h_{1}(x)=S \psi(x) \text { for a.e. } x \in M,
$$

and let $\zeta$ be the $h_{1}$-invariant partition of $M$, induced by $\psi$. It follows from proposition 1.1 and lemma 3.1 that there are $D \subset M, h_{1} D=D, \mu(D)=1, U \subset M / \zeta, h_{1}^{\zeta} U=U$, $\mu_{\zeta}(U)=1$ and an integer $n>0$ such that for every $C \in U$ the intersection $C \cap D$ consists of exactly $n$ points each of $\mu_{C}$-measure $1 / n$.

We assume without loss of generality that $D=M$ and $U=M / \zeta$. So each $C \in \zeta$ consists of $n$ distinct points of $\mu_{C}$-measure $1 / n$.

Let $\theta, K, \rho, K^{\prime}, \varepsilon$ and $\delta_{0}$ be as in $\S 4$ for $\zeta$.

We omit the proof of the following lemma, since it is fully analogous to the proof of lemma 4.1 and corollary 4.1 .

Lemma 5.1. Let $0<\delta<\delta_{0}, u, v \in M$ and let

$$
\begin{aligned}
A_{k}= & \{m \in\{0,1, \ldots, k\}: \text { there exists } v(m) \in \zeta(v) \\
& \text { such that } \left.h_{m} v(m) \in K^{\prime} \text { and } d\left(h_{m} u, h_{m} v(m)\right)<\delta\right\} .
\end{aligned}
$$

If $\left|A_{k}\right| / k>0.9$ for all integers $k>k_{0}>0$ then there is $\tilde{v} \in \zeta(v)$ such that

$$
\tilde{v}=h_{q} u \text { for some } q=q(u, v, \delta),|q|<\delta .
$$

Let $X<\zeta, \mu(X)=1$ and $X_{i} \subset X, i=1, \ldots, n$.

$$
\begin{gathered}
X_{i} \cap X_{i}=\varnothing, \quad i \neq j, \\
\bigcup_{i=1}^{n} X_{i}=X, \quad \mu\left(X_{i}\right)=\frac{1}{n}, \quad i=1, \ldots, n
\end{gathered}
$$

be such that for every $x \in X$ the intersection $\zeta(x) \cap X_{i}$ consists of a single point $x_{i}(x)$ and the map $\phi_{i}: X$ onto $X_{i}$ defined by $\phi(x)=x_{i}(x)$, is measurable.

As in $\S 4$ we denote

pick

$$
\tilde{K}=K^{\prime} \cap X, \quad \tilde{K}<\zeta, \quad \mu(\tilde{K})=\mu\left(K^{\prime}\right)>1-\theta / n,
$$

$$
\Lambda \subset X, \quad \Lambda<\zeta, \quad \mu(\Lambda)>1-\theta
$$


such that each $\phi_{i}, i=1, \ldots$, is uniformly continuous on $\Lambda$ and take

$$
Q=\Lambda \cap \tilde{K}, \quad \mu(Q)>1-2 \theta, \quad Q<\zeta .
$$

Let $F \subset M$ be the generic set of $Q$ for $h_{1}$. We have

$$
h_{1} F=F, \quad F<\zeta \quad \text { and } \mu(F)=1 .
$$

LEMMA 5.2. For every $0<\delta<\delta_{0}$ there is $\beta=\beta(\delta)$ such that if $u_{1}, v_{1} \in F, v_{1}=h_{1} u_{1}$ for some $|t|<\beta$ then for every $u_{2} \in \zeta\left(u_{1}\right)$ there is $v_{2} \in \zeta\left(v_{1}\right)$ such that $v_{2}=h_{a} u_{2}$ for some $a=a\left(u_{1}, u_{2}, t\right),|a|<\delta$ and $a\left(h_{1} u_{1}, h_{1} u_{2}, t\right)=a\left(u_{1}, u_{2}, t\right)$.

Proof. The proof is similar to that of lemma 4.2. Let $\beta>0$ be such that

$$
\begin{aligned}
& \text { if } d\left(w_{1}, w_{2}\right)<\beta, w_{1}, w_{2} \in \Lambda \text { then } \\
& \quad d\left(\phi_{i} w_{1}, \phi_{i} w_{2}\right)<\delta, \quad i=1, \ldots, n .
\end{aligned}
$$

Let

$$
u_{1}, v_{1} \in F \quad \text { and } \quad v_{1}=h_{t} u_{1} \quad \text { for some }|t|<\beta .
$$

Since $u_{1}, v_{1} \in F$ there is $k_{0}>0$ such that if $k \geq k_{0}$ and

$$
B_{k}=\left\{m \in\{0,1, \ldots, k\}: h_{m} u_{1} \in Q, h_{m} v_{1} \in Q\right\}
$$

then

$$
\left|B_{k}\right| / k>1-7 \theta
$$

where $|B|$ denotes the number of points in $B$.

Let $u_{2} \in \zeta\left(u_{1}\right)$. We write $j(m)=i \in\{1, \ldots, n\}$ if $h_{m} u_{2} \in X_{i}, m=1,2, \ldots$ We have

$$
\begin{gathered}
\phi_{j(m)}\left(h_{m} u_{1}\right)=h_{m} u_{2} \in X_{j(m)} \\
\phi_{j(m)}\left(h_{m} v_{1}\right) \in \zeta\left(h_{m} v_{1}\right)=h_{m} \zeta\left(v_{1}\right)
\end{gathered}
$$

or

$$
\phi_{j(m)}\left(h_{m} v_{1}\right)=h_{m} v_{1}(m)
$$

for some $v_{1}(m) \in \zeta\left(v_{1}\right)$ and if $m \in B_{k}$ then

$$
h_{m} u_{2} \in K^{\prime}, \quad h_{m} v_{1}(m) \in K^{\prime}
$$

and

$$
d\left(h_{m} u_{2}, h_{m} v_{1}(m)\right)<\delta
$$

by (5.1). This and (5.2) imply via lemma 5.1 that there is $v_{2} \in \zeta\left(v_{1}\right)$ such that

$$
v_{2}=h_{a} u_{2}
$$

for some $a=a\left(u_{1}, u_{2} t\right),|a|<\delta$. It is clear that

$$
a\left(h_{1} u_{1}, h_{1} u_{2}, t\right)=a\left(u_{1}, u_{2}, t\right) .
$$

Let $T(x)$ denote the $h_{t}$-orbit of $x \in M$ and let

$$
\bar{F}=\{x \in M: F \cap T(x) \text { is dense in } T(x)\} .
$$

$\bar{F}$ is $h_{t}$-invariant, $t \in R$ and $\mu(\bar{F})=1$.

It follows from lemma 5.2 that if $x \in \bar{F}, x_{i} \in T(x) \cap F, i=1,2, \ldots$ and $x_{i} \rightarrow x, i \rightarrow \infty$ in the topology of $T(x)$ then the $\lim _{i \rightarrow \infty} \zeta\left(x_{i}\right)$ exists and does not depend on the sequence $x_{i} \in T(x) \cap F, x_{i} \rightarrow x, i \rightarrow \infty$. If $x \in \bar{F} \cap F$ then this limit equals to $\zeta(x)$. 
We define $\bar{\zeta}$ on $\bar{F}$ by

$$
\bar{\zeta}(x)=\zeta(x) \text { if } x \in \bar{F} \cap F
$$

and

$$
\bar{\zeta}(x)=\lim _{i \rightarrow \infty} \zeta\left(x_{i}\right) \quad \text { if } x \in \bar{F}-F
$$

where $x_{i} \in T(x) \cap F, i=1,2, \ldots$ and $x_{i} \rightarrow x, i \rightarrow \infty$ in $T(x)$.

$\bar{\zeta}$ is well defined and

$$
\bar{\zeta}(x)=\zeta(x) \text { for a.e. } x \in M .
$$

Proof of theorem 3. In order to prove the theorem it is enough to show that there exists an $h_{t}$-invariant set

$$
F^{\prime} \subset \bar{F}, \quad \mu\left(F^{\prime}\right)=1, \quad F^{\prime}<\zeta
$$

such that

$$
h_{t}(\bar{\zeta}(x))=\bar{\zeta}\left(h_{t} x\right) \quad \text { for all } x \in F^{\prime} \text { and all } t \in R \text {. }
$$

It follows from lemma 5.2 that for every $x \in \bar{F}, \tilde{x} \in \bar{\zeta}(x)$ and $t \in R$ there is $a=a(x, \tilde{x}, t) \in R$ such that

$$
\begin{gathered}
h_{a} \tilde{x} \in \bar{\zeta}\left(h_{t} x\right) \\
a\left(h_{1} x, h_{1} \tilde{x}, t\right)=a(x, \tilde{x}, t) \\
a(x, x, t)=t, \quad a(x, \tilde{x}, 0)=0, \quad a(x, \tilde{x}, 1)=1 .
\end{gathered}
$$

The function $a(x, \tilde{x}, t)$ is uniformly continuous in $t$ for every $x \in \bar{F}, \tilde{x} \in \bar{\zeta}(x)$.

Denote

$$
\begin{aligned}
& r^{-}(x, t)=\min \{a(x, \tilde{x}, t): \tilde{x} \in \bar{\zeta}(x)\} \\
& r^{+}(x, t)=\max \{a(x, \tilde{x}, t): \tilde{x} \in \bar{\zeta}(x)\}, x \in \bar{F}, t \in R .
\end{aligned}
$$

$r^{-}(x, t)$ and $r^{+}(x, t)$ are continuous in $t$ and are constant on the $h_{1}$-orbit of $x$. Since $h_{1}$ is ergodic, there is $F_{t} \subset \bar{F}, F_{t}<\bar{\zeta}, h_{1} F_{t}=F_{t}, \mu\left(F_{t}\right)=1$ such that $r^{+}(x, t)$ and $r^{-}(x, t)$ equal constants $r^{+}(t)$ and $r^{-}(t)$ respectively on $F_{t}$.

Let

$$
\tilde{F}=\bigcap_{t \text { is rational }} F_{t}, \quad \mu(\tilde{F})=1, \quad h_{1} \tilde{F}=\tilde{F}, \quad \tilde{F}<\bar{\zeta}
$$

We have

$$
\begin{aligned}
& r^{-}(x, t)=r^{-}(t) \\
& r^{+}(x, t)=r^{+}(t)
\end{aligned}
$$

for every $x \in \tilde{F}$ and every rational $t$. Since $r^{+}(x, t)$ and $r^{-}(x, t)$ are continuous in $t$, (5.4) holds for all $t \in R$.

Let

$$
F^{\prime}=\{x \in \bar{F}: \tilde{F} \cap T(x) \text { is dense in } T(x)\},
$$

$h_{\uparrow} F^{\prime}=F^{\prime}, t \in R, F^{\prime}<\bar{\zeta}$ and $\mu\left(F^{\prime}\right)=1$. (5.4) implies that

$$
r^{-}(x, t)=r^{-}(t), \quad r^{+}(x, t)=r^{+}(t)
$$


for all $x \in F^{\prime}$ and all $t \in R$, since

$$
r^{+}(x, t)=\lim _{i \rightarrow \infty} r^{+}\left(x_{i}, t\right), r^{-}(x, t)=\lim _{i \rightarrow \infty} r^{-}\left(x_{i}, t\right)
$$

if $x_{i} \in T(x) \cap \tilde{F}$ and $x_{i} \rightarrow x$ in $T(x)$.

Take $x \in F^{\prime}$ and let $\tilde{x} \in \bar{\zeta}(x)$ be such that

$$
h_{r^{-}(t)} \tilde{x} \in \bar{\zeta}\left(h_{t} x\right)
$$

We have

$$
a(x, \bar{x}, t) \geq r^{-}(t)=a(x, \tilde{x}, t) \quad \text { for every } \bar{x} \in \bar{\zeta}(x) .
$$

This implies that

$$
a\left(\tilde{x}, \bar{x}, r^{-}(t)\right) \geq r^{-}(t) \text { for all } \bar{x} \in \bar{\zeta}(x)
$$

and therefore

$$
r^{-}\left(r^{-}(t)\right)=r^{-}(t) \quad \text { for all } t \in R
$$

We claim that

$$
r^{-}(t)=r^{+}(t)=t \quad \text { for all } t \in R .
$$

Indeed, it follows from (5.3) and the definition of $r^{+}$and $r^{-}$that

and

$$
\begin{aligned}
& r^{-}(0)=r^{+}(0)=0 \\
& r^{-}(1)=r^{+}(1)=1
\end{aligned}
$$

$$
r^{-}(t)+r^{+}(1-t)=1
$$

Let us first show that

$$
r^{-}\left(\frac{1}{2}\right)=r^{+}\left(\frac{1}{2}\right)=\frac{1}{2} .
$$

Since $r^{-}(t)$ is continuous, there is $t_{0} \in(0,1)$ such that

$$
r^{-}\left(t_{0}\right)=\frac{1}{2}
$$

This and (5.5) imply that

$$
r^{-}\left(\frac{1}{2}\right)=\frac{1}{2}
$$

and therefore

$$
r^{+}\left(\frac{1}{2}\right)=\frac{1}{2}
$$

by (5.7). We have shown that if $x \in F^{\prime}$ then

This implies that

$$
h_{1 / 2} \bar{\zeta}(x)=\bar{\zeta}\left(h_{1 / 2} x\right)
$$

$$
r^{-}(t)+r^{+}\left(\frac{1}{2}-t\right)=\frac{1}{2} \quad \text { for all } t \in R .
$$

Arguing as above we get that (5.6) holds for $t=\frac{1}{4}$ and $t=\frac{3}{4}$. Proceeding by induction, we get that (5.6) holds for all $t \in R$ of the form $k / 2^{n}, k, n=1,2, \ldots$ Since $r^{-}$and $r^{+}$are continuous, (5.6) holds for all $t \in R$. (5.6) implies that

$$
h_{t} \bar{\zeta}(x)=\bar{\zeta}\left(h_{t} x\right) \text { for all } x \in F^{\prime} \text { and all } t \in R \text {. }
$$

This completes the proof.

This work was partially supported by NSF grant MCS-74-19388. 


\section{REFERENCES}

[1] B. Marcus. The horocycle flow is mixing of all degrees. Inventiones Math. 46 (1978), 201-209.

[2] D. Ornstein \& B. Weiss. Geodesic flows are Bernoullian. Israel J. Math. 14 (1973), 184-197.

[3] M. Ratner. Horocycle flows are loosely Bernoulli. Israel J. Math. 31 (1978), 122-131.

[4] M. Ratner. Rigidity of horocycle flows. Ann. Math. 115 (1982), 597-614.

[5] V. A. Rohlin. On basic concepts of measure theory. Mat. Sbornik 67 (1949), 107-150. (In Russian.)

[6] H. C. Wang. On a maximality property of discrete subgroups with fundamental domain of finite measure. Amer. J. Math. 89 (1967), 124-132. 